\title{
THE ORIGIN OF DENDROSENECIO WITHIN THE SENECIONEAE (ASTERACEAE) BASED ON CHLOROPLAST DNA EVIDENCE ${ }^{1}$
}

\author{
ERIC B. KNOX ${ }^{2,3}$ AND JefFrey D. PALMER \\ Herbarium and Department of Biology, University of Michigan, Ann Arbor, Michigan 48109-1048; and \\ Department of Biology, Indiana University, Bloomington, Indiana 47405
}

\begin{abstract}
Chloroplast DNA restriction-site variation was surveyed using 15 enzymes for 37 accessions from the tribe Senecioneae (Asteraceae), plus two outgroup species, in order to determine the placement within the tribe of the giant senecios (Dendrosenecio). The survey revealed 176 phylogenetically informative mutations and 121 autapomorphic mutations. Dendrosenecio is diagnosed by a minimum of 15 mutations, which suggests that the giant senecios evolved from a relatively isolated lineage within the Senecioneae, and this conclusion is supported by earlier evidence from chromosome counts and phytochemistry. Among the taxa sampled, the closest relatives of Dendrosenecio are Cineraria deltoidea and two species of Euryops. Support was not found for suggestions in the literature that the closest relatives of Dendrosenecio are species in Solanecio or Senecio subgen. 'Crociseris.' The position of the Dendrosenecio/Cineraria/Euryops clade is weakly supported as basal to the majority of other senecionoid genera. The tussilaginoid genera sampled (Ligularia, Petasites, Roldana, and Tussilago) are monophyletic in our analysis, with the surprising inclusion of Pericallis hybrida as the sister-taxon to Roldana suffulta. The sister-group to the Dendrosenecio/CinerarialEuryops clade includes all species of Delairea, Gynura, Kleinia, Packera, Senecio, and Solanecio sampled. Within Senecio, subgenera Senecio and 'Crociseris' form a monophyletic core, with subgenus 'Kleinioidei' being broadly paraphyletic or possibly polyphyletic.
\end{abstract}

Since the discovery of Dendrosenecio johnstonii (Oliv.) B. Nord. (Asteraceae) on Mt. Kilimanjaro, Tanzania, by H. H. Johnston (1886), the giant senecios have attracted considerable interest and discussion by taxonomists and evolutionary biologists. These plants typically have large leaf-rosettes borne on thick, woody stems that branch after flowering to produce candalabra-like growth forms the size of telephone poles. They have diversified on ten mountains in eastern Africa (Knox, 1993a; Knox and Palmer, 1995) to form 17 taxa (11 species and six nonautonymic infraspecific taxa; Knox, 1993b), and the adaptive radiation of this group involved much convergent or parallel evolution, resulting in a "mosaic of variation" in morphological features (Mabberley, 1973) that confounded earlier attempts to reconstruct their evolutionary history. The giant senecios were initially treated as Senecio (Oliver, 1887), a placement explicitly supported by several workers (Cotton, 1932, 1944; Hedberg, 1957; Mabberley, 1973, 1974) until Nordenstam (1978) recognized Dendrosenecio as a genus distinct from a more narrowly delimited Senecio (Knox, 1993b).

A recurrent question in the literature concerns the clos-

\footnotetext{
${ }^{1}$ Manuscript received 16 December 1994; revision accepted 23 May 1995.

The authors thank the Tanzanian Commission for Science and Technology, the Institute Zairois pour la Conservation de la Nature, the Office Rwandais du Tourisme et des Parcs Nationaux, and Addis Abeba University and the Ethiopian Ministry for Foreign Affairs for permission to conduct research in their respective countries; $R$. K. Jansen for DNA and helpful comments on the manuscript; and M. C. Burd, R. R. Kowal, the Missouri Botanical Garden, and the Royal Botanic Gardens, Kew for seed or leaf tissue. This work was supported in part by grants from the National Science Foundation (BSR-8800487, BSR-8901123, BSR-8996262) and awards from the Rackham School of Graduate Studies, The University of Michigan.

${ }^{2}$ Present address: Department of Biological Sciences, Rutgers University, Newark, NJ 07102.

${ }^{3}$ Author for correspondence (Fax: 201-648-1007).
}

est relatives of the giant senecios. Several species in $\mathrm{Se}$ necio subgen. 'Crociseris' (see Table 1 for authors) and the segregate genus Solanecio have been suggested. Within Senecio, these include S. subsessilis (Engler, 1892 ) and other coarse herbs from upland eastern Africa in sect. Plantaginei (S. ochrocarpus and S. karaguensis O. Hoffm.; Mabberley, 1974; Nordenstam, 1978), the coarse European herbs $S$. umbrosus and $S$. doronicum in sect. Crociseris (Mabberley, 1974), and the South American S. hualtata DC. in sect. Hualtatini (DC.) Bak. (Hauman, 1935). The two Solanecio species are robust shrubs from tropical Africa (S. mannii; Carlquist, 1974, p.554) and Ethiopia, (S. gigas; Nordenstam, 1978). With the exception of Senecio karaguensis and $S$. hualtata, the remaining species were included in a preliminary survey (Knox, 1993c) of restriction-site variation, which included the North American herb Packera aurea and used the cultivar Pericallis hybrida (formerly treated as Cineraria; Jansen et al., 1990; Jansen, Michaels, and Palmer, 1991) as the outgroup species. This survey found that these putative close relatives to Dendrosenecio shared a more recent common ancestry with each other, and that Pericallis, Dendrosenecio, and the clade comprising all other taxa sampled were separated by long basal branches. In the present study, this preliminary survey of the tribe Senecioneae was therefore expanded using readily available material (Table 1) in order to determine the placement of Dendrosenecio within the tribe and to identify potential close relatives.

In the most recent treatment of the Senecioneae, Jeffrey (1992) accepted the two subtribes (Blennospermatinae and Senecioninae) delimited by Nordenstam (1977) and proposed many provisional infrasubtribal groupings that were not formally described. His stated purpose was to provide a list of putative monophyletic genera (and subordinate groups within the still heterogeneous genus 


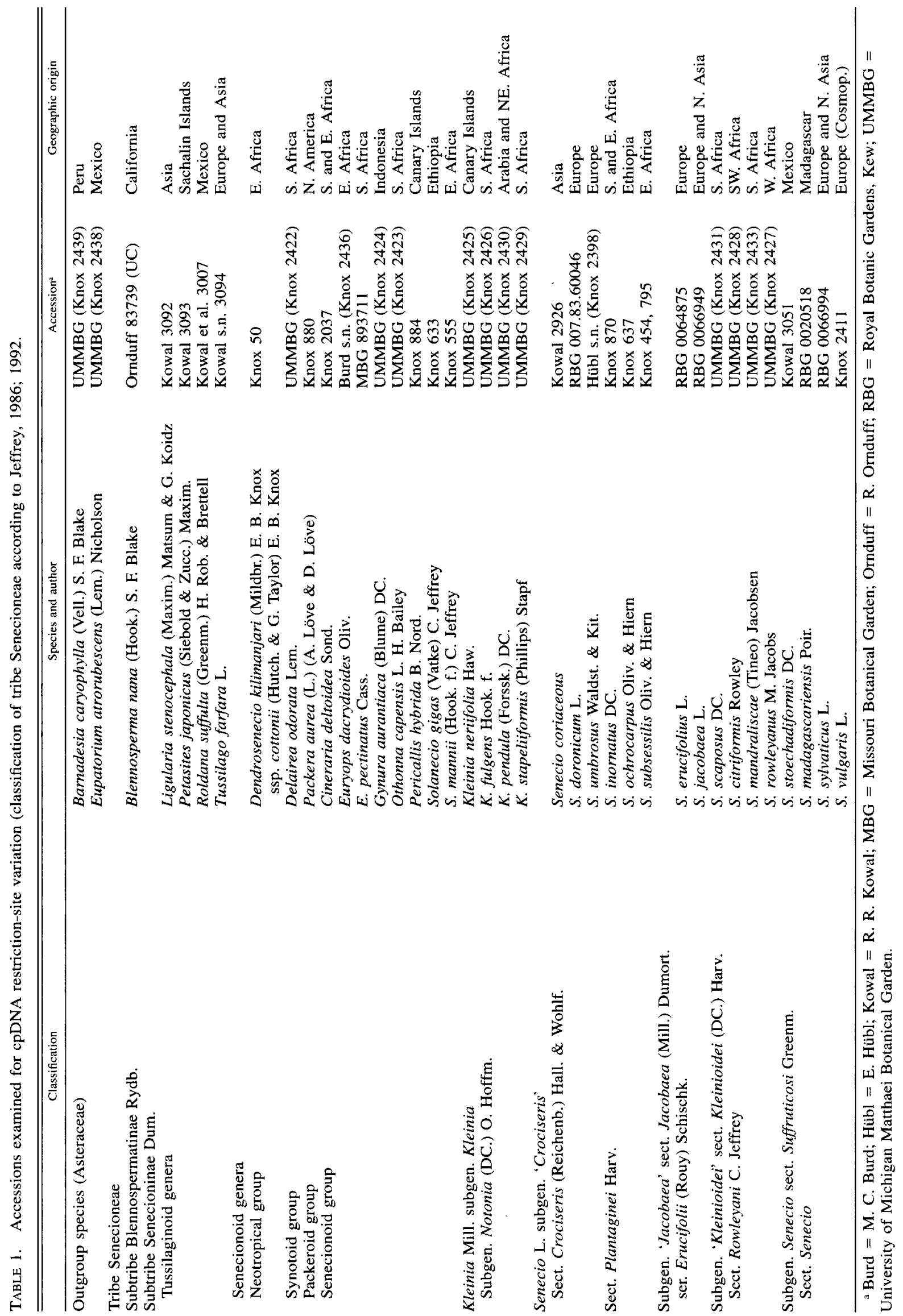


Senecio) for the selection of representative species in future systematic studies. We have used Jeffrey's informal classification for the species included in our survey (Table 1), but our sampling represents only a small part of the 112 genera, comprising an estimated 3,400 species, in the tribe. It was not our intention to evaluate Jeffrey's treatment, but our results provide some preliminary insight into infratribal relationships.

\section{MATERIALS AND METHODS}

Plant material was obtained from various sources (Table 1) either as seed or fresh or dried leaf tissue. Within the subtribe Senecioninae, we included 32 accessions representing 11 senecionoid genera (Cineraria, Delairea, Dendrosenecio, Euryops, Gynura, Kleinia, Othonna, Packera, Pericallis, Senecio, and Solanecio) and one accession from each of four tussilaginoid genera (Ligularia, Petasites, Roldana, and Tussilago). Blennosperma nana was used to represent the subtribe Blennospermatinae, and Barnadesia caryophylla and Eupatorium atrorubescens were used as ultimate outgroup species (Jansen and Palmer, 1987; Jansen et al., 1990; Jansen, Michaels, and Palmer, 1991). Voucher specimens are deposited in the University of Michigan Herbarium (MICH) or the University of Wisconsin Herbarium (WIS; Kowal Collections). Dendrosenecio kilimanjari subsp. cottonii was used as a single placeholder to represent the Dendrosenecio clade for the reasons given in the Results.

Total cellular DNA was isolated using the modified CTAB procedure of Doyle and Doyle (1987), and was purified by centrifugation in cesium chloride/ethidium bromide gradients. DNA aliquots from all samples were digested with each of 15 restriction enzymes: AseI, BamHI, BanI, BanII, BclI, BglII, BstNI, ClaI, DdeI, DraI, EcoO109, EcoRI, EcoRV, HindIII, and NciI. Twenty-two clones of a lettuce cpDNA library (Jansen and Palmer, 1987, 1988; Knox, Downie, and Palmer, 1993) were used as probes in filter-hybridization experiments to map restriction sites in the cpDNAs. Two subclones from tobacco (Solclones 2 and 3 in Olmstead and Palmer, 1992) were used as probes to represent the one uncloned portion of the lettuce genome. Methods were performed as described in Palmer (1986), Palmer et al. (1988), and Knox, Downie, and Palmer (1993). Gel electrophoresis for DNA digested with DdeI, which cuts cpDNA frequently, was conducted using a $2 \%$ gel in order to provide better resolution of small fragments.

Restriction-site maps were constructed for all enzymes except DdeI. For this enzyme, only two unambiguous, phylogenetically informative restriction sites were included in the data set. All Senecioneae have two derived inversions relative to tobacco (Jansen and Palmer, 1987; Knox, Downie, and Palmer, 1993). Barnadesia caryophylla lacks these inversions, and only unambiguous, shared mutations were scored for $B$. caryophylla and the other outgroup species, Eupatorium atrorubescens. For the ingroup species, autapomorphies were included in the data set for all enzymes except $D d e l$. Presence or absence of a restriction site was coded as 1 or 0 , respectively, thereby eliminating the need for polarity assignments prior to analysis using outgroup comparison (Watrous and Wheeler, 1981). The analyses, which included bootstrap and decay analyses (Felsenstein, 1985; Bremer, 1988; Donoghue et al., 1992), were conducted using PAUP (Swofford, 1993) on a Macintosh computer. An heuristic search with the Random Addition option (100 replicates) was performed to ensure that all islands of shortest trees were found (Maddison, 1991).

\section{RESULTS}

Our survey of restriction-site variation revealed 176 phylogenetically informative restriction-site mutations and 121 autapomorphic mutations out of a total of $\approx 720$ sites surveyed in each genome (excluding the DdeI sites that were not mapped; data matrix available from E. B. Knox upon request). A phylogenetic analysis of the 176 shared mutations found a single island of eight shortest trees of 349 steps, with a consistency index (CI; Kluge and Farris, 1969) of 0.50. The eight shortest trees result from alternative topologies for the clade comprising $\mathrm{Se}$ necio subgen. Senecio and subgen. 'Crociseris.' The strict consensus tree with Barnadesia and Eupatorium as the outgroup is shown in Fig. 1. We have indicated the minimum number of restriction-site mutations that support each node, which we feel is a more conservative way of reporting the results than the convential way of selecting either the acctran or deltran optimization of character support. Our analysis of 40 accessions that represent all 11 species of Dendrosenecio shows remarkably little cpDNA divergence (only nine variable sites; Knox and Palmer, 1995). In order to limit computational demands for bootstrap and decay analyses, we have used a single placeholder for the genus. Of these nine variable sites, five are unique and one is homoplastic within Dendrosenecio, while the remaining three are homoplastic with taxa outside Dendrosenecio. Because of the large number of mutations that diagnose Dendrosenecio (a minimum of 15 , with seven of these unique mutations), our results are unaffected by the choice of a placeholder, and we have accordingly used $D$. kilimanjari subsp. cottonii, which is basal within the genus and has no mutations of its own.

Of the 15 unambiguously diagnostic restriction-site mutations for Dendrosenecio, seven are unique restriction-site gains and the remaining eight (seven site losses and one gain) are homoplastic within the context of this data set but are consistently optimized as diagnostic for Dendrosenecio. Three additional mutations may also be diagnostic, but the pattern of homoplasy does not permit unambiguous character optimization for the Euryops/CinerarialDendrosenecio clade. The placement of Cineraria deltoidea as the closest relative to Dendrosenecio has a moderate level of support (bootstrap $=67 \%$, decay $=2$ ). The placement of the two Euryops species as the sistergroup of CinerarialDendrosenecio is less strongly supported (bootstrap $=49 \%$, decay $=1$ ).

\section{DISCUSSION}

The origin of Dendrosenecio-Chloroplast DNA evidence indicates that those robust herbaceous and shrubby species from Senecio and Solanecio that we have sampled and that were previously suggested as close relatives to Dendrosenecio are all more closely related to each other than any are to Dendrosenecio. The giant senecios, apparently decaploid $(n=50)$; show virtually no meiotic irregularities that might suggest a recent origin from the predominantly tetraploid $(n=20)$ putatively close relatives suggested in the literature (Knox and Kowal, 1993). Dendrosenecio lacks the furoeremophilanes typically found in Senecio, Euryops, Ligularia, and Othonna, and contains $p$-hydroxyacetophenone derivatives and scopoletin, which are rare in Senecio, and resorcinols that have been isolated in the Asteraceae only from Baccharis quitensis HBK (tribe Astereae; Dupré, Bohlmann, and Knox, 1990). The prenylated p-hydroxyphenones are more abundant in the tribes Eupatorieae and Heliantheae, and it is of interest to note that the main constituent (the methyl ether of 2-senecioyl-p-hydroxyacetophenone) was 


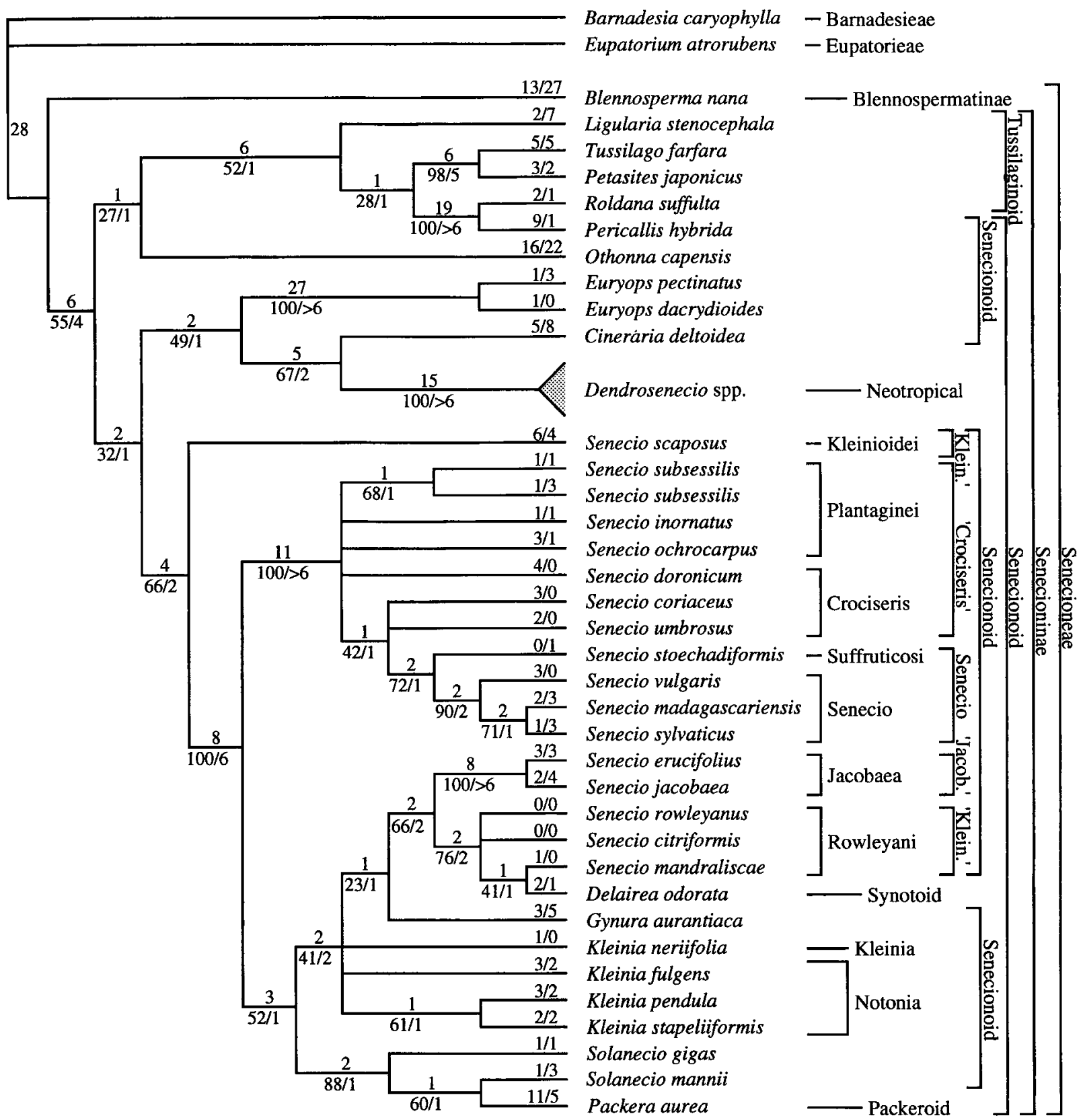

Fig. 1. Wagner parsimony tree for 37 accessions of Senecioneae with Barnadesia and Eupatorium used as outgroup species. This is a strict consensus of eight equally parsimonious trees generated using 176 synapomorphic restriction-site mutations. Each tree has 349 steps and a CI of 0.50. The numbers above the line at each node indicate the minimum branch length. These values were taken from the table of linkages in the tree description output of PAUP and do not depend on whether ACCTRAN, DELTRAN, or MINF optimization is selected. The reporting of minimum lengths avoids the potentially arbitrary selection of a particular optimization method, but as a consequence, the sum of internal branch lengths and homoplastic changes ( 289 steps; see tree) will be less than the total tree length ( 349 steps). The numbers below the branches and preceding the slashes are percentage values that indicate the number of times that a monophyletic group occurred in 100 bootstrap replicates. The numbers after these slashes are decay values that indicate the number of steps needed for a clade to lose resolution. The numbers before and after the slash above the terminal lines indicate the minimum number of homoplastic changes and autapomorphies, respectively. Bootstrap and decay values for the Dendrosenecio clade are based on a more detailed study of this group (E. B. Knox and J. D. Palmer, unpublished data).

originally isolated from Espeletia schultzii Wedd. (Bohlmann and Rao, 1973), an Andean alpine species with many convergent adaptations of the giant-rosette growth form seen in Dendrosenecio (Hedberg and Hedberg, 1979). The high chromosome number (with no irregularities) and the unusual phytochemistry support the conclu- sion that the lineage that gave rise to Dendrosenecio may be relatively ancient and isolated within the Senecioneae, although Dendrosenecio itself seems to have had a relatively recent origin ( 1 million yr) and rapid radiation in eastern Africa (Knox and Palmer, 1995). A similar pattern of a recent radiation of a lineage separated from its 
closest relatives by a long branch length was recently reported in Aquilegia (Ranunculaceae; Hodges and Arnold, 1994).

It is important to keep in mind that the molecular phylogenetic basis of our conclusion that Dendrosenecio is an isolated lineage within the Senecioneae is a function of our sampling strategy, which was to search among all potential (and also accessible) closest relatives of this genus. The large number of restriction-site mutations (15) separating Dendrosenecio from Cineraria is significant in ways that the large numbers of mutations separating, for example, Euryopys (27) or the Senecio subsessilissylvaticus clade (11) from their closest examined relatives are not. If we had been looking for the closest relatives of Euroyops or this Senecio clade, and our sampling had exhausted all likely possibilities (as it did for Dendrosenecio), then we could have drawn similar conclusions about these taxa. In fact, we feel confident that we could locate species that are basal to our pair of Euryops species or this Senecio clad, and hence start breaking up these long branches, but at this point it is not obvious where we should look for closer relatives of the giant senecios. We are not saying that these other long branches are not potentially significant; rather, our sampling (or lack thereof) does not allow us to interpret these branch lengths in the same way that we can interpret the results with respect to Dendrosenecio.

Cineraria deltoidea is a small, straggling, semiwoody climber, which in eastern Africa grows primarily in open montane forest but occasionally reaches the alpine zone $(3,750 \mathrm{~m})$. Euryops pectinatus is a fast-growing south African shrub commonly planted as an ornamental that has escaped into disturbed, midaltitude sites (1,750-2,250 $\mathrm{m}$ ) in Kenya. Euryops dacrydioides is an endemic alpine shrub on Mt. Kilimanjaro. The basal positions of Cineraria and Euryops relative to the Dendrosenecio clade suggest that the ancestor of Dendrosenecio was at least semiwoody and may have been frost tolerant, but few other conclusions can be drawn because of the length of the branch separating Dendrosenecio and Cineraria and the numerous morphological differences among Dendrosenecio, Cineraria, and Euryops.

Although the ancestor of the giant senecios remains undetermined, some possibilities can be eliminated. In much the same way that the east African mountains can be thought of as a two-dimensional array of habitat islands (E. B. Knox and J. D. Palmer, unpublished data), the question of ancestral relations for Dendrosenecio can be conceptualized using three dimensions, namely, altitude, latitude, and longitude. In the global pattern of vegetation, the latitudinal ecological continua (e.g., Arctic to north temperate alpine to tropical alpine to south temperate alpine to Antarctic) have an obvious altitudinal correlation. Longitude represents a third, idiosyncratic dimension that may facilitate or hinder the geographic movement of plants (e.g., the circumboreal landmasses vs. the oceans, lowlands, and mountains of the tropics).

Because none of the previously suggested east African candidates proved to be close relatives, the recent origin of Dendrosenecio via a simple altitudinal event can be eliminated as a likely explanation. Previous arguments against this possibility have stressed the problem of acquiring frost tolerance (Carlquist, 1974, pp. 550, 569). It seems doubly unlikely that Dendrosenecio originated from frost-intolerant plants growing elsewhere in the tropics (i.e., via a combined altitudinal and longitudinal event). The close relationship between the species in Senecio subgen. 'Crociseris' sect. Crociseris (Europe) and sect. Plantaginei (Africa) suggests that other temperate candidates in subgen. 'Crociseris' are not likely to be closely related to Dendrosenecio. This eliminates a simple northto-south latitudinal event as a likely explanation.

The remaining possibilities include: (1) a simple longitudinal event of dispersal from tropical highlands elsewhere in the world; (2) a simple south-to-north latitudinal event from a south African ancestor; and (3) a combined latitudinal/longitudinal event, which merely has a longer dispersal distance than a simple latitudinal event. From an ecological standpoint, the first possibility is most attractive because colonists from an ancestor growing in tropical highlands elsewhere would have the greatest preadaptation for establishment on the mountains in eastern Africa. On the other hand, there are no obvious close relatives to Dendrosenecio based on growth form and gross morphology, so either extensive modification occurred during the origin of Dendrosenecio, or close relatives with similar modifications have gone extinct. The emerging picture of Dendrosenecio as a recent diversification of a relatively old lineage suggests that close relatives should be sought among species that are regarded as relictual. Additional sampling from tropical highland species in the so-called neotropical group of senecionoid genera (Jeffrey, 1992) with a chromosome number of 50 (Knox and Kowal, 1993) may reveal closer relatives of Dendrosenecio than Cineraria, and the 15 unambiguously diagnostic restriction-site mutations for Dendrosenecio provide a firm empirical basis for screening potential relatives (information on genomic location of and restriction enzyme used for each variable site is available from $\mathrm{E}$. B. Knox),

The tribe Senecioneae-Although this survey was not designed to provide definitive insight into the phylogenetic relationships within the Senecioneae, some provisional conclusions can be drawn. The sister-group relationship between subtribe Senecioninae and our single representative of subtribe Blennospermatinae (Blennosperma nana; Fig. 1) supports the earlier conclusions of Bremer (1987) and Jansen, Michaels, and Palmer (1991) concerning the basal division within the Senecioneae. Within the subtribe Senecioninae, the tussilaginoid genera have weak support (bootstrap $=51 \%$, decay $=1$ ) as a monophyletic group, with the surprising inclusion of Pericallis hybrida, which is strongly supported as a close relative of Roldana suffulta. Nordenstam (1978) regarded Pericallis as "a very natural and homogeneous group, which is difficult to relate to any extant portion of Senecio s. str. ...' Both Pericallis and Roldana have chromosome numbers of $n=30$ (Nordenstam, 1978; Jeffrey, 1992) and also share the trait of sub-palmately lobed and veined leaves (R. R. Kowal, personal communication), and four of the Pericallis species were previously placed in the tussilaginoid genus Doronicum (Nordenstam, 1978).

The clearly basal position of the succulent Senecio scaposus within the Delairea/Gynura/Kleinia/Packera/Senecio/Solanecio clade (Fig. 1) indicates that the succulent Senecio subgen. 'Kleinioidei' is not a monophyletic 
group, but additional sampling is needed in order to determine whether the succulent senecios represent a broadly paraphyletic assemblage or, more likely, are the result of convergent evolution. Our sampling indicates that $\boldsymbol{S e}$ necio subgen. Senecio is a monophyletic group that is nested within the paraphyletic subgen. 'Crociseris'. These two subgenera probably form the core of a monophyletic Senecio.

Our three samples of sect. Rowleyani constitute a clade that includes the anomalous Delairea odorata (syn. S. mikanioides Otto ex Walp.). The deeply nested position of Delairea suggests that the features linking it with genera in the synotoid group are the result of convergence, but additional sampling is needed to draw firm conclusions. The sister-group of sect. Rowleyani is evidently Senecio subgen. 'Jacobaea' sect. Jacobaea ser. Erucifolii, which is monophyletic based on our sampling. It is likely that subgen. 'Kleinioidei' (excluding S. scaposus and its relatives) and subgen. 'Jacobaea' have retained primitive features from the ancestral Senecio, and that the lineage giving rise to these subgenera has also spawned the segregate genera Gynura, Kleinia, Packera, and Solanecio, but, again, more sampling is needed.

The unresolved topology involving Kleinia is due to a lack of variation, and Kleinia is possibly monophyletic. The position of Gynura aurantiaca is only weakly supported, and may prove to be more basal with additional sampling. The weakly supported position of Packera aurea as the sister-species (within our survey) of Solanecio mannii (which makes Solanecio paraphyletic) is unexpected and suggests a need for greater taxonomic and molecular sampling for these two genera.

Although this survey provides a preliminary framework for considering phylogenetic relationships within the Senecioneae, much more work is needed in order to obtain a sufficiently reliable and detailed phylogeny on which to base taxonomic decisions. (More extensive molecular surveys of the Senecioneae are being conducted by J. W. Kadereit [Mainz, Germany; in conjunction with C. Jeffreyl, and anyone willing to contribute material for molecular systematic studies in the Senecioneae should contact Dr. Kadereit.) One clear result is that none of the putative close relatives previously suggested in the literature are in fact closely related to Dendrosenecio. In order to reconstruct the morphological features of the progenitor of Dendrosenecio and to determine whether the features formerly used to place Dendrosenecio within $\mathrm{Se}$ necio are retained primitive features or the result of convergent evolution, closer relatives must be found, and these relatives are not likely to be in Senecio sensu stricto or its closely allied genera.

NOTE ADDED IN PROOF: The correct identification of the species listed as "Euryops pectinatus Cass." in Table 1 and Fig. 1 is "Euryops chrysanthemoides (DC.) B. Nord."

\section{LITERATURE CITED}

Bohlmann, F., and N. RaO. 1973. Neue hydroxyacetophenone-derivate aus Espeletia schultzii Wedd. Chemische Berichte 106: 3035-3038.
BREMER, K. 1987. Tribal relationships of the Asteraceae. Cladistics 3: $210-253$.

1988. The limits of amino acid sequence data in angiosperm phylogenetic reconstruction. Evolution 42: 795-803.

Carlquist, S. 1974. Island biology. Columbia University Press, New York, NY.

CotTon, A. D. 1932. The tree senecios of the African mountains. Proceedings of the Linnean Society of London 1931-1932: 110-111.

- 1944. The megaphytic habit in the tree senecios and other genera. Proceedings of the Linnean Society of London, Session 156: $158-168$.

Donoghue, M. J., R. G. Olmstead, J. F. Smith, and J. D. Palmer. 1992. Phylogenetic relationships of Dipsacales based on $r b c \mathrm{~L}$ sequences. Annals of the Missouri Botanical Garden 79: 333-345.

DoYLE, J. J., AND J. L. DoYLE. 1987. A rapid DNA isolation for small quantities of fresh tissue. Phytochemical Bulletin of the Botanical Society of America 19: 11-15.

Dupré, S., F. Bohlmann, And E. Knox. 1990. Prenylated p-hydroxyacetophenone derivatives from the giant Senecio johnstonii. Biochemical Systematics and Ecology 18: 149-150.

ENGLeR, A. 1892. Über die Hochgebirgsflora des tropischen Afrika. Abhandlungen der Königlichen Akademie der Wissenschaften zu Berlin 1891: 1-461.

FElsenstein, J. 1985. Confidence limits on phylogenies: an approach using the bootstrap. Evolution 39: 783-791.

HaUman, L. 1935. Les Senecio arborescents du Congo. Revue de Zoologie et de Botanique Africaines 28: 1-76.

HedBerg, I., AND O. HedBerg. 1979. Tropical-alpine life-forms of vascular plants. Oikos 33: 297-307.

HEDBERG, O. 1957. Afroalpine vascular plants: a taxonomic revision. Symbolae Botanicae Upsalienses 15: 1-411.

Hodges, S. A., AND M. L. ARnold. 1994. Columbines: a geographically widespread species flock. Proceedings of the National Academy of Sciences, USA 91: 5129-5132.

Jansen, R. K., K. E. Holsinger, H. J. Michaels, and J. D. Palmer. 1990. Phylogenetic analysis of chloroplast DNA restriction site data at higher taxonomic levels: an example from the Asteraceae. Evolution 44: 2089-2105.

, H. J. Michaels, AND J. D. Palmer. 1991. Phylogeny and character evolution in the Asteraceac based on chloroplast DNA restriction site mapping. Systematic Botany 16: 98-115.

, AND J. D. PALMER. 1987. A chloroplast DNA inversion marks an ancient evolutionary split in the sunflower family (Asteraceae). Proceedings of the National Academy of Sciences, USA 84: 58185822.

-, AND - 1988. Phylogenetic implications of chloroplast DNA restriction site variation in the Mutisieae (Asteraceae). American Journal of Botany 75: 753-766.

Jeffrey, C. 1986. The Senecioneae in east tropical Africa. Notes on Compositae: IV. Kew Bulletin 41: 873-943.

- 1992. The tribe Senecioneae (Compositae) in the Mascarene Islands with an annotated world check-list of the genera of the tribe. Notes on Compositae: VI. Kew Bulletin 47: 49-109.

Johnston, H. H. 1886. The Kilima-Njaro expedition, a record of scientific exploration in East Equatorial Africa. K. Paul, Trench, \& Co., London.

Kluge, A. G., AND J. S. Farris. 1969. Quantitative phyletics and the evolution of anurans. Systematic Zoology 18: 1-32.

KNOX, E. B. 1993a. The conservation status of the giant senecios and lobelias in eastern Africa. Opera Botanica 121: 195-216.

. 1993b. The species of giant senecio (Compositae) and giant lobelia (Lobeliaceae) in eastern Africa. Contributions from the University of Michigan Herbarium 19: 241-257.

. 1993c. Evolution of the giant senecios and giant lobelias in eastern Africa. Ph.D. dissertation, University of Michigan. Ann Arbor, MI.

- S. R. Downie, and J. D. Palmer. 1993. Chloroplast genome rearrangements and the evolution of giant lobelias from herbaceous ancestors. Molecular Biology and Evolution 10: 414-430.

- AND R. R. Kowal. 1993. Chromosome numbers of the east African giant senecios and giant lobelias and their evolutionary significance. American Journal of Botany 80: 847-853.

, And J. D. PAlmer. 1995. Chloroplast DNA variation and the 
recent radiation of the giant senecios (Asteraceae) on the tall mountains of eastern Africa. Proceedings of the National Academy of Sciences, USA 92: 10349-10353.

Mabberley, D. J. 1973. Evolution of the giant groundsels. Kew Bulletin 28: 61-96.

. 1974. Branching in pachycaul senecios: the Durian Theory and the evolution of angiospermous trees and herbs. New Phytologist 73: 967-975.

MADDISON, D. R. 1991. The discovery and importance of multiple islands of most-parsimonious trees. Systematic Zoology 40: 315-328.

Nordenstam, B. 1977. Senecioneae and Liabeae-systematic review. In V. H. Heywood, J. B. Harborne, and B. L. Turner [eds.], The biology and chemistry of the Compositae, 799-830. Academic Press, New York, NY.

1978. Taxonomic studies in the tribe Senecioneae (Compositae). Opera Botanica 44: 1-84.
OLIVER, D. 1887. The plants of the Kilima-Njaro expedition. Transactions of the Linnean Society, London, ser. 2, 2: 327-355.

Olmstead, R. G., AND J. D. Palmer. 1992. A chloroplast DNA phylogeny of the Solanaceae: subfamilial relationships and character evolution. Annals of the Missouri Botanical Garden 79: 346-360.

PALMER, J. D. 1986. Isolation and structural analysis of chloroplast DNA. Methods in Enzymology 118: 167-186.

, R. K. Jansen, H. J. Michaels, M. W. Chase, and J. R. ManHART. 1988. Chloroplast DNA variation and plant phylogeny. Annals of the Missouri Botanical Garden 75: 1180-1206.

SwOFFORD, D. L. 1993. PAUP: phylogenetic analysis using parsimony, version 3.1.1. Smithsonian Institution, Washington, DC.

Watrous, L., AND Q. WhEELER. 1981. The out-group comparison method of character analysis. Systematic Zoology 30: 1-11. 